\title{
Mucoadhesive liposomal delivery systems: the choice of coating material
}

\author{
Pankaj Ranjan Karn ${ }^{1,2}$, Zeljka Vanić ${ }^{3}$, Ivan Pepic ${ }^{3}$ and Nataša Škalko-Basnet ${ }^{1,4}$ \\ ${ }^{1}$ The School of Pharmaceutical and Biomedical Sciences, Pokhara University, Lekhnath, Nepal, ${ }^{2}$ Laboratory of \\ Pharmaceutical Engineering, Collage of Pharmacy, Chungnam National University, Daejeon, Korea, ${ }^{3}$ Department of \\ Pharmaceutics, Faculty of Pharmacy and Biochemistry, University of Zagreb, Zagreb, Croatia and ${ }^{4}$ Department of \\ Pharmacy, Drug Transport and Delivery Research Group, University of Tromsø, Tromsø, Norway
}

\begin{abstract}
Objective: Development of liposomal mucoadhesive drug delivery system, which is able to improve the bioavailability of poorly absorbed oral drugs by prolonging their gastric and intestinal residence time, through facilitating the intimate contact of the delivery system with the absorption membrane. Materials and methods: Liposomes containing model drug atenolol were prepared by the modified ethanol injection method. Liposomes containing atenolol were coated by different mucoadhesive polymers, for example, chitosan, Carbopol 974P, Eudragit L100, and Eudragit S100, to optimize the choice of coating material. The delivery systems were tested for their in vitro mucoadhesiveness. Results: Liposomes prepared by the ethanol injection method were of satisfactory size (around $100 \mathrm{~nm}$, before coating). Through the coating of liposomes in the presence of unentrapped material, the entrapment efficiency for drug was increased. In vitro mucoadhesive test confirmed the mucoadhesive properties of the coated layer for all tested polymers; however, Eudragit S100-coated liposomes were superior to other coating materials. Discussion: Eudragit coating appeared to be an optimal polymer choice. These preliminary data indicate that polymer-coated mucoadhesive liposomes are able to carry sufficient amount of drug and to remain attached to the intestinal mucosa for a sufficient period of time to enable prolonged absorption of entrapped drug. Conclusion: While keeping in mind that the in vivo conditions may vary with the in vitro ones, we may recommend the system described in our work for possible oral delivery of peptides and phytochemicals.
\end{abstract}

Key words: Atenolol, Carbopol, chitosan, Eudragit, liposomes, mucoadhesion, polymer-coating

\section{Introduction}

With the rapid progress in pharmaceutical technology, delivery of therapeutic proteins/peptides has received a considerable amount of attention over the past 10 years for potential clinical applications ${ }^{1,2}$. The oral route remains to be the most convenient and comfortable way of drug administration, including peptide delivery. However, peptide drugs are readily degraded under the low $\mathrm{pH}$ of the gastric medium and by various proteolytic enzymes in the gastrointestinal (GI) tract $^{3}$. To improve the oral absorption efficiencies, multifunctional polymers exhibiting mucoadhesive, pH-dependent, permeation-enhancing, enzyme inhibitory properties have been used ${ }^{4,5}$.
One of the most promising strategies in developing mucoadhesive particulate systems is surface modification, or coating, of the drug carrier particles with mucoadhesive polymers ${ }^{5,6}$. Liposomes were proposed as suitable drug delivery systems for various types of drug molecules, including proteins and peptides ${ }^{7}$. Liposomes possess the advantages that they are composed of physiological materials, for example, phospholipid, but are liable to be destructed by the $\mathrm{pH}$, bile salts, and pancreatic lipase in the GI tract. Apart from these major barriers, fragile nature and short in vitro half-lives of these bioactives preclude many options to formulate an oral dosage form. To minimize the destructive influences, the formation of a polymeric membrane around the liposome has been proposed $^{8,9}$. 
Mucoadhesive dosage forms have received substantial attention as novel drug delivery systems able to improve the bioavailability of drugs by prolonging their residence time and controlling the drug release characteristics. Mucoadhesive nanoparticulate systems such as polymercoated liposomes were found to be useful carriers for improved oral delivery because of their prolonged retention in the GI tract and excellent penetration into the mucus layer ${ }^{5}$. The mucoadhesive liposomes can be prepared by coating drug carrier surface by various mucoadhesive polymers such as chitosan ${ }^{10-13}$, Carbopol ${ }^{9,14,15}$, and Eudragit ${ }^{16}$. Chitosan \{poly [ $\beta$-(1-4)-2-amino-2-deoxyD-glucopyranose] $\}$ is the second abundant natural-origin polysaccharide next to cellulose and, over the past two decades, has been used for various biomedical and drug delivery applications because of its low toxicity, good biocompatibility, and antimicrobial and mucoadhesive properties. Its mucoadhesive properties are due to the molecular attractive forces formed by the electrostatic interaction between positively charged chitosan and negatively charged mucosal surfaces ${ }^{17,18}$. Carbopol polymers are polymers of acrylic acid cross-linked with polyalkenyl ethers or divinyl glycol and they are also finding numerous applications in oral mucoadhesive drug delivery because of their ability to interact with the mucus glycoprotein and to remain localized to a specific site $^{14,15,19}$. Polymethacrylates for pharmaceutical purposes became known under the trademark Eudragit. Eudragit L100 is an anionic polymer synthesized from methacrylic acid and methacrylic acid methyl ester and has a pH-dependent solubility. It is slowly soluble in intestinal fluid that is mildly acidic to neutral. Eudragit L100, as an enteric coating material, is soluble above $\mathrm{pH}$ 6.0 medium, which can protect the protein drugs from the acidic environment in the stomach ${ }^{20}$. Eudragit S100 is an anionic polymer synthesized from methacrylic acid and methacrylic acid methyl ester and has a $\mathrm{pH}$-dependent solubility. It is slowly soluble in the region of GI tract where juices are neutral to weakly alkaline ${ }^{21}$.

By the right combination of liposomal and polymer characteristics, it is possible to develop delivery system with specific, prolonged, and controlled release ${ }^{22}$.

The objective of this work was to optimize the mucoadhesive liposomal delivery system for oral administration of peptides and possibly phytochemicals by the optimization of coating mucoadhesive material. The vesicle size before and after coating, entrapment efficiencies for model drug atenolol, and the mucoadhesive strength of the delivery systems based on chitosan, Carbopol, and Eudragit coatings were used as parameters in evaluating the optimal formulation.

\section{Materials and methods}

\section{Materials}

Eudragit S100 and Eudragit L100 (Rohm GmbH \& Co. KG, Darmstad, Germany) were kindly provided by LS
Pharmazeutishe Technologie, Pharmazeutishes Institut, Freiburg University, Germany. Carbopol 974P (BF Goodrich, Cleveland, OH, USA) and atenolol (Atenolol IP, IPCA Laboratories, Mumbai, India) were generous gifts of Quest Pharmaceuticals, Birganj, Nepal. Chitosan (LMW) was purchased from Sigma (Tokyo, Japan). Lecithin was from Siegfried AG (Zofingen, Germany). Lipoid S100 and Lipoid S75 were generous gifts from Lipoid GmbH (Ludwigshafen, Germany). The other chemical reagents and solvents used were of analytical grade. Pig intestinal membrane from freshly slaughtered animal was purchased from the local slaughterhouse.

\section{High-performance liquid chromatography analysis of atenolol}

The analyses were performed on a Shimadzu chromatographic system equipped with high-performance liquid chromatography (HPLC) Shimadzu pump LC-20AD, a Shimadzu SPD-20A UV spectrophotometer. (Tokyo, Japan) The column $\mathrm{RP}_{18}$ Fluofix 3NW415 $(4.60 \times 150 \mathrm{~mm})$ was joined with precolumn. The mobile phase consisted of a mixture of acetonitrile/ methanol/0.01 $\mathrm{M}$ phosphate buffer with $\mathrm{pH}$ adjusted to 6.0 with $0.1 \mathrm{M} \mathrm{NaOH}$, containing $0.1 \%$ sodium dodecyl sulfate (34:33:33, v/v/v), pumped at a flow rate of $1.0 \mathrm{~mL} / \mathrm{min}^{23}$. The eluting compounds were monitored at UV $258 \mathrm{~nm}$ and temperature of the column was maintained at $30^{\circ} \mathrm{C}$ during the chromatographic separation. The retention time of the atenolol was determined to be 5.3 minutes.

\section{Preparation of liposomes}

Liposomes were prepared by the modified ethanol injection method ${ }^{24}$. Three types of lipids, namely, Lipoid S100, Lipoid S75, and lecithin, were used in the preparations (Table 1). Lipid was dissolved in absolute ethanol $(25-100 \mathrm{mg} / \mathrm{mL})$ mixed with either ethanol or the ethanolic solution of atenolol $(10-30 \mathrm{mg} / \mathrm{mL})$ and $1.5 \mathrm{~mL}$ of such a mixed solution rapidly injected into $20 \mathrm{~mL}$ of magnetically stirred phosphate buffer saline (PBS; $\mathrm{pH}$ 7.4; Table 1). Stirring was continued for 1 hour and the liposomal suspension was left overnight in refrigerator to stabilize before characterization. The final ethanol concentration $(7.5 \%)$ and the rate of stirring were kept constant for all preparations.

\section{Coating of liposomes Chitosan coating}

For the preparation of chitosan-coated liposomes, $0.1 \%$ and $0.6 \%(\mathrm{w} / \mathrm{v})$ chitosan solutions were prepared in $0.1 \%$ $(\mathrm{v} / \mathrm{v})$ glacial acetic $\mathrm{acid}^{25}$. A volume of $2.0 \mathrm{~mL}$ of chitosan solution was added drop-wise to the $2.0 \mathrm{~mL}$ of liposomes under magnetic stirring at room temperature for 1 hour, followed by incubation in refrigerator overnight. The rate of stirring was kept constant for all preparations by this method. 
Table 1. Composition of uncoated and coated liposomal formulations.

\begin{tabular}{|c|c|c|c|c|c|c|c|c|}
\hline \multirow[b]{2}{*}{$\begin{array}{l}\text { Formula- } \\
\text { tion code }\end{array}$} & \multicolumn{8}{|c|}{ Composition } \\
\hline & $\begin{array}{l}\text { Atenolol } \\
(\mathrm{mg})\end{array}$ & $\begin{array}{l}\text { Lecithin } \\
\text { (mg) }\end{array}$ & $\begin{array}{l}\text { Lipoid S75 } \\
\text { (mg) }\end{array}$ & $\begin{array}{l}\text { Lipoid S100 } \\
\text { (mg) }\end{array}$ & $\begin{array}{l}\text { Chitosan } \\
(w / w)(\%)\end{array}$ & $\begin{array}{c}\text { Carbopol } 940 \\
(\mathrm{w} / \mathrm{w})(\%)\end{array}$ & $\begin{array}{l}\text { Eudragit L100 } \\
(\mathrm{w} / \mathrm{w})(\%)\end{array}$ & $\begin{array}{c}\text { Eudragit S100 } \\
(\mathrm{w} / \mathrm{w})(\%)\end{array}$ \\
\hline$\overline{\text { EL1 }}$ & & 20 & & & & & & \\
\hline EL2 & & & 20 & & & & & \\
\hline EL3 & & & & 20 & & & & \\
\hline ATL1 & 6.6 & 20 & & & & & & \\
\hline ATL2 & 6.6 & & 20 & & & & & \\
\hline ATL3 & 6.6 & & & 20 & & & & \\
\hline ELCh01 & & & & 200 & 0.1 & & & \\
\hline ELCh06 & & & & 20 & 0.6 & & & \\
\hline ELCa01 & & & & 20 & & 0.1 & & \\
\hline ELCa06 & & & & 20 & & 0.6 & & \\
\hline ELEuL01 & & & & 20 & & & 0.1 & \\
\hline ELEuL06 & & & & 20 & & & 0.6 & \\
\hline ELEuS01 & & & & 20 & & & & 0.1 \\
\hline ELEuS06 & & & & 20 & & & & 0.6 \\
\hline ATLCh01 & 6.6 & & & 20 & 0.1 & & & \\
\hline ATLCh06 & 6.6 & & & 20 & 0.6 & & & \\
\hline ATLCa01 & 6.6 & & & 20 & & 0.1 & & \\
\hline ATLCa06 & 6.6 & & & 20 & & 0.6 & & \\
\hline ATLEuL01 & 6.6 & & & 20 & & & 0.1 & \\
\hline ATLEuL06 & 6.6 & & & 20 & & & 0.6 & \\
\hline ATLEuS01 & 6.6 & & & 20 & & & & 0.1 \\
\hline ATLEuS06 & 6.6 & & & 20 & & & & $0-6$ \\
\hline
\end{tabular}

EL, empty liposomes; ATL, liposomes containing atenolol, uncoated; ATLCh, ATL coated with chitosan; ATLCa, ATL coated with Carbopol 940; ATLEuL, ATL coated with Eudragit L100; ATLEuS, ATL coated with Eudragit S100.

\section{Carbopol coating}

For the preparation of Carbopol-coated liposomes, 0.1\% and $0.6 \%(\mathrm{w} / \mathrm{v})$ Carbomer 974P was dissolved in PBS 7.4. A volume of $2.0 \mathrm{~mL}$ of Carbopol solution was added drop-wise to the $2.0 \mathrm{~mL}$ of liposomes under magnetic stirring at room temperature for 1 hour, followed by incubation in refrigerator overnight. The rate of stirring was kept constant for all preparations by this method.

\section{Eudragit coating}

For the Eudragit coating two types of Eudragit, namely, Eudragit S100 and Eudragit L100 were used. The method applied was the modification of the method used for chitosan coating. Eudragits were dissolved in acetone containing $3.0 \%$ of water and drop-wise added to liposomes and left overnight in refrigerator to stabilize before characterization.

\section{Particle size determination}

Hydrodynamic diameter $S\left(d_{\mathrm{h}}\right)$ of liposomes and polymercoated liposomes was measured by dynamic light scattering (DLS) with a vertically polarized He-Ne laser beam at a wavelength of $633 \mathrm{~nm}$ (Zetasizer $3000 \mathrm{HS}$, Malvern Instruments, Malvern, UK). The scattering angle was fixed at $90^{\circ}$ and the temperature was maintained at $25^{\circ} \mathrm{C}$. The samples were filtered through $1.20-\mu \mathrm{m}$ membrane before measurements (Chromafil ${ }^{\circledR}$ MachereyNagel, Detlef Lambrecht, Germany). Analyses were made using the CONTIN algorithm.

\section{Zeta potential determination}

The formation of chitosan layer on liposomal surface was confirmed by measuring the zeta potential on vesicle surface. Zeta potential of the uncoated and chitosan-coated $(0.6 \%, \mathrm{w} / \mathrm{v})$ vesicles was determined using a Zetasizer Nano Z (Malvern Instruments). Three measurements of the two samples for each type of vesicles were analyzed.

\section{Entrapment efficiency determination}

Dialysis was applied to separate unentrapped atenolol from liposomes or polymer-coated liposomes ${ }^{10}$. The liposomal samples $(4.0 \mathrm{~mL})$ and the polymer-coated liposomal samples $(3.0 \mathrm{~mL})$ were placed in tubing and extensively dialyzed against the PBS 7.4 for 24 hours. The dialysis was continued for 48 hours in total. The volume of buffer was adjusted so that the concentration of drug in the buffer was kept below the solubility of drug. The drug concentration (unentrapped) was determined spectrophotometrically.

After removal of unentrapped material, liposomes were destroyed using methanol and the actual content of drug in the sample was determined by the HPLC method. For this, $500 \mu \mathrm{L}$ of liposomal suspension was mixed thoroughly with $2.0 \mathrm{~mL}$ of methanol and the solution was filtered through the hexamethylenediamine, nylon-66 membrane filter of pore size $0.20 \mu \mathrm{m}$. For the coated liposomes, the destruction was done using the methanol and 
chloroform in the ratio of 2:1. A volume of $20 \mu \mathrm{L}$ of clear solution was injected and the concentration of drug was calculated directly by measuring the peak area through the standard calibration curve of atenolol.

\section{In vitro wash-off test for mucoadhesive testing}

The mucoadhesive property of the polymer-coated liposomes was evaluated by an in vitro adhesion test. The method used was the modified in vitro wash-off test ${ }^{26}$. The mucoadhesion of the polymer-coated liposomes was compared with that of a nonmucoadhesive material, uncoated liposomes containing atenolol. Freshly excised pieces of pig intestinal mucosa $(2 \times 2 \mathrm{~cm})$ were tightened onto glass slides $(3 \times 1$ inches $)$ with thread. A volume of $500 \mu \mathrm{L}$ of the liposomes, $0.6 \%$ (w/v) chitosan-coated liposomes, $0.6 \%(\mathrm{w} / \mathrm{v})$ Carbopol-coated liposomes, $0.6 \%(\mathrm{w} /$ v) Eudragit S100, and 0.6\% (w/v) Eudragit L100-coated liposomes were spread onto each wet-rinsed tissue specimen and immediately incubated at $37^{\circ} \mathrm{C}$. The tissue specimens were taken out at 1 and 3 hours. The samples were washed with $10.0 \mathrm{~mL}$ of PBS at each time interval. The results were evaluated based on the $t$-test comparison between uncoated and polymer-coated liposomes.

\section{Determination of mucoadhesive strength}

From the $10.0 \mathrm{~mL}$ of the eluted buffer containing nonadhered drug, $500 \mu \mathrm{L}$ aliquots were taken and liposomal lipids were dissolved by methanol. From the clear solution, a volume of $20 \mu \mathrm{L}$ was injected and the peak area was measured by HPLC.

The concentration of atenolol eluted in the buffer (PBS 7.4) was measured and the remaining drug was assumed to be present in liposomes adhered to the intestinal mucosa. Hence, the percentage of mucoadhesive strength can be calculated by Equation (1)

$$
\begin{aligned}
& \text { Mucoadhesion }(\%)= \\
& {\left[\frac{\text { Amount of drug remaining in mucosa }}{\text { Amount of drug taken in test }}\right] \times 100 \text {. }}
\end{aligned}
$$

\section{Results and discussion}

\section{Atenolol determination}

HPLC analysis confirmed single peak of atenolol (Figure 1) with the linearity of the standard curve obtained in the range of $10-100 \mu \mathrm{g} / \mathrm{mL}$ resulting in the correlation coefficient of 0.9998 .

\section{Particle size analysis \\ Comparison of particle size of empty liposomes, liposomes containing drug, and polymer-coated liposomes containing drug}

Particle size of vesicles affects the biological properties of delivery system. The mucoadhesiveness of the coated liposomes was reported to be dependent on the particle size, and submicron-sized chitosan-coated liposomes were found to be more effective than the chitosancoated liposomes of bigger size. The mucoadhesive property of smaller-size chitosan-coated liposomes is the result of chitosan coating attributing to the longer retention in the intestinal tract ${ }^{27}$.

As the first step in developing mucoadhesive liposomes of desired particle size distribution, we examined the effect of the purity of phospholipids used for liposomal preparation. The liposomal size and polydispersity indexes, as determined by photon correlation spectroscopy, revealed rather large particles with broad size distribution in the case of liposomes made of lecithin even before the actual coating procedure (Table 2); thereof, we decided to use further only pure phosphatidylcholine such as Lipoid S100. Moreover, polydispersity indexes for liposomes containing atenolol prepared from Lipoid S100 revealed more uniform size distributions with lower polydispersity index. During the size distribution measurements by the DLS method, it was observed that the mean diameter of the whole population was affected by a very small population of relatively larger vesicles which contributed to increased polydispersity index. Therefore, we used membrane filter with pore size of $1.20 \mu \mathrm{m}$ to filter liposomal samples before measurement

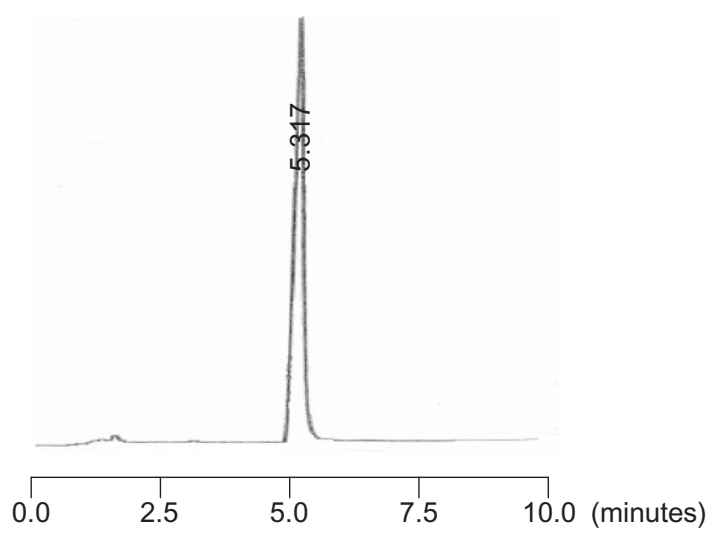

Figure 1. Typical HPLC chromatogram of standard atenolol. Chromatographic conditions: column: Fluofix $(4.6 \times 150 \mathrm{~mm})$; column temperature: $30^{\circ} \mathrm{C}$; mobile phase: acetonitrile : methanol: $0.01 \mathrm{M}$ phosphate buffer $\mathrm{pH} 6.0$ (34:33:33); detector $\mathrm{UV}$ at $258 \mathrm{~nm}$; flow rate: $1 \mathrm{~mL} / \mathrm{min}$; chart speed: $5 \mathrm{~mm} / \mathrm{min}$; and retention time: 5.3 minutes.

Table 2. Particle size analysis of liposomes prepared by the ethanol injection method varying phospholipid purity.

\begin{tabular}{llccc}
\hline $\begin{array}{l}\text { Preparation } \\
\text { code }\end{array}$ & $\begin{array}{c}\text { Type of } \\
\text { lipid used }\end{array}$ & $\begin{array}{c}\text { Drug } \\
(\mathrm{mg})\end{array}$ & $\begin{array}{c}\text { Mean } \\
\text { diameter }\end{array}$ & $\begin{array}{c}\text { Polydispersity } \\
(\mathrm{nm})\end{array}$ \\
\hline EL1 & Lecithin & - & $241.3 \pm 17.6$ & 0.890 \\
EL2 & Lipoid S75 & - & $184.2 \pm 20.3$ & 0.699 \\
EL3 & Lipoid S100 & - & $97.7 \pm 1.51$ & 0.365 \\
ATL1 & Lecithin & 6.66 & $281.6 \pm 5.9$ & 0.760 \\
ATL2 & Lipoid S75 & 6.66 & $167.2 \pm 14.0$ & 0.672 \\
ATL3 & Lipoid S100 & 6.66 & $89.0 \pm 3.5$ & 0.223 \\
\hline
\end{tabular}

The amount of lipid used in all formulations was $20 \mathrm{mg}$. The values denote the mean \pm SD of three separate set of experiments. 
to eliminate this small population of larger vesicles or agglomerates. After this intervention, the mean size of liposomes did not change significantly, but the polydispersity index was reduced. During the filtration, less than 5\% of original total amount of lipid was lost (data not shown) as determined by the phospholipid assay.

The ethanol injection method is one of the simplest liposomal preparation methods. Liposomes prepared by this method are expected to be of $100 \mathrm{~nm}$ in diameter. In our results, liposomes with model drug have particle size below $100 \mathrm{~nm}$ (Figure 2). The mean diameter of empty liposomes was $97.7 \mathrm{~nm}$, whereas liposomes containing atenolol were $89.0 \mathrm{~nm}$ in size. Similar results were reported by Filipovic-Grcic et al. ${ }^{10}$ The particle size of polymer-coated liposomes in each formulation has increased with the increasing concentration of polymer solution used in the coating, suggesting the formation of coating layer on the surface of the liposomes and an increase in thickness of the coating layer with an increase in the concentration of polymer solution as observed by Filipovic-Grcic et al. ${ }^{10}$, Wu et al. ${ }^{28}$, and Shende and Gaud ${ }^{29}$. By the increase in the concentration of polymer used for coating, the increase of the mean particle size was observed, however, the reason for the increase in size might not be only the consequence of the formation of coating layer on liposomal surface, but possible agglomerates of two or more vesicles stacked together, which cannot be distinguished from this measurement method.

In addition, the zeta potential measurements of uncoated and chitosan-coated $(0.6 \%, \mathrm{w} / \mathrm{v})$ vesicles confirmed the presence of polymer layer on liposomal surface. The uncoated liposomes had zeta potential of $-20 \pm$ $3 \mathrm{mV}$, whereas chitosan-coated liposomes had zeta potential of $27 \pm 6 \mathrm{mV}$. The increase in zeta potential values for chitosan-coated liposomes is in agreement with Takeuchi and yamamoto. ${ }^{5}$

\section{Entrapment efficiency for atenolol in uncoated and polymer-coated liposomes}

The physicochemical properties of drug, especially its solubility and partition coefficient, can be important determinants for the extent of its liposomal incorporation. The degree or efficiency of drug entrapment in liposomes, assessed as entrapment yield, depends on the number of factors, including drug's characteristics, liposomal size, and liposomal composition ${ }^{24}$. The entrapment efficiency for atenolol was calculated by HPLC external standard method (Table 3, Figure 1). The amount and the type of lipid were kept constant. The efficiencies were found to vary and to be rather low. It is probably the consequence of the hydrophilicity of drug $(\log P=0.16)$ and smaller liposomal size.

Table 3. Entrapment efficiency for polymer-coated liposomes containing atenolol.

\begin{tabular}{lcc}
\hline $\begin{array}{l}\text { Type of polymer } \\
\text { used in coating }\end{array}$ & $\begin{array}{c}\text { Concentration } \\
\text { of polymer (\%) }\end{array}$ & $\begin{array}{c}\text { Entrapment } \\
\text { efficiency (\%) }\end{array}$ \\
\hline- & - & $21.62 \pm 0.92$ \\
Chitosan & 0.1 & $24.68 \pm 3.51$ \\
Chitosan & 0.6 & $25.77 \pm 0.29$ \\
Carbopol & 0.1 & $22.19 \pm 5.69$ \\
Carbopol & 0.6 & $22.22 \pm 0.33$ \\
Eudragit L100 & 0.1 & $26.09 \pm 1.32$ \\
Eudragit L100 & 0.6 & $32.93 \pm 0.06$ \\
Eudragit S100 & 0.1 & $31.53 \pm 2.21$ \\
Eudragit S100 & 0.6 & $43.57 \pm 0.51$ \\
\hline
\end{tabular}

Liposomes were prepared by the modified ethanol injection method and coated in the presence of unentrapped material. The values denote the mean of three separate sets of experiments \pm SD.

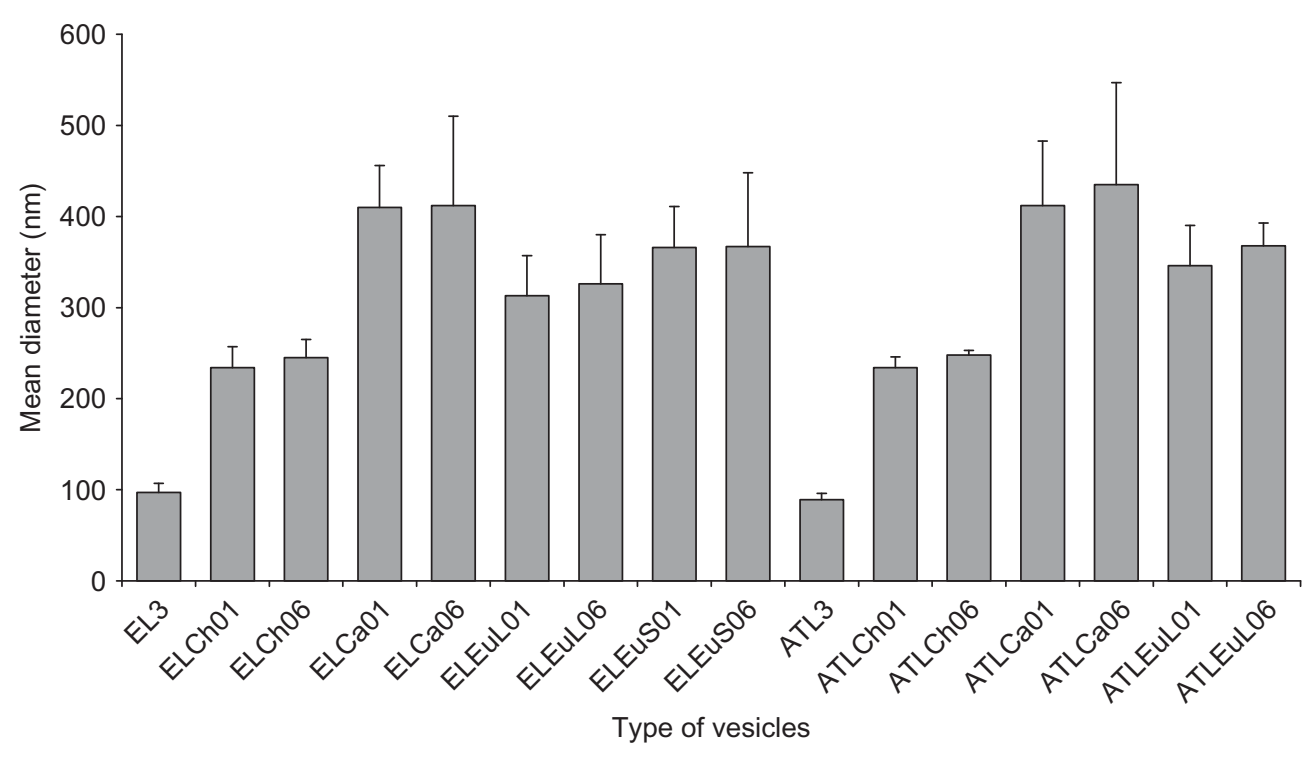

Figure 2. Particle size distribution of coated liposomes. The lipid used in all preparations was Lipoid S100. The values denote the mean \pm SD of three separate sets of experiments. 


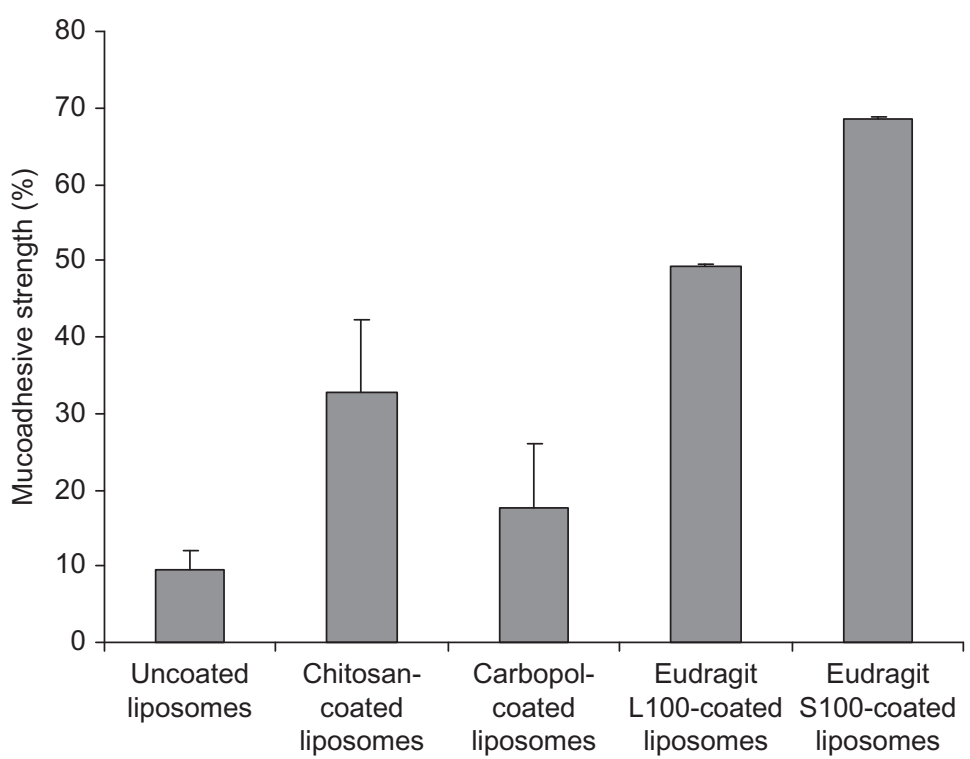

Figure 3. Mucoadhesiveness of polymer-coated liposomes. Liposomes containing atenolol were prepared by the modified ethanol injection method and coated by various polymers by procedures explained earlier. The mucoadhesiveness was tested by the modified wash-off method. The results represent mean of three separate sets of experiments.

The entrapment efficiency for atenolol was remarkably increased after coating in the presence of unentrapped atenolol, particularly in the case of Eudragit coating (Table 3), suggesting that mucoadhesive coating may have application for peptide and protein drugs delivery ${ }^{10}$.

The entrapment efficiency was found to be higher in all cases of polymer-coating of liposomes, but remarkable results were obtained in the case of Eudragit L100and Eudragit S100-coated liposomes. The 0.6\% (w/v) Eudragit S100 increases the entrapment efficiency by $110 \%$, whereas $0.6 \%(\mathrm{w} / \mathrm{v})$ Eudragit L100 increases it about 55\%. Both Eudragit S100 and Eudragit L100 are the anionic polymers of methacrylic acid with known superiority in the intestinal drug delivery systems. These polymers would be the suitable carrier for the possible oral peptide or phytochemical drug delivery systems $^{20,21}$.

Based on our preliminary results, we suggest that by the right choice of polymer used in coating, and depending on the characteristics of the drug to be entrapped in liposomes, polymer-coated liposomes may encapsulate sufficient amount of drug, and be a promising tool in oral delivery of various molecules.

\section{In vitro mucoadhesion testing of polymer-coated liposomes}

The in vitro test of mucoadhesion was performed by the modified wash-off method on pork intestine. The percentage of mucoadhesive strengths was calculated by Equation (1) and the results demonstrated that the polymer-coated liposomes have higher strength compared with the nonmucoadhesive material $(P<0.05)$. After 3 hours of incubation, more than $68 \%$ of the originally entrapped atenolol was retained on the intestinal mucosa in the case of Eudragit S100-coated liposomes containing atenolol (Figure 3). Both Eudragit L100- and Eudragit S100-coated liposomes were found to possess stronger mucoadhesive properties compared with chitosan-coated and particularly Carbopol-coated liposomes.

The preliminary results presented in Figure 3 confirm the possibility of using mucoadhesive polymer-coated liposomes as a promising tool in oral delivery of various molecules. One should keep in mind that in vivo situation may not directly follow the in vitro findings, because in vivo conditions include the GI motility and peristaltic movements which, in our experimental conditions, were not present. However, the adhesive strength of $68 \%$ observed in the present experiment might contribute to significant mucoadhesion in in vivo conditions. When deciding on the choice of coating material, several parameters contribute to the decision. Based on the drug entrapment efficiency and mucoadhesive strength, it appears that Eudragit-coated liposomes possess superior properties to chitosan- and Carbopol-coated liposomes. The decision will be effected by the short and long stability of liposomal systems and the possibility for scale up of the manufacturing process.

\section{Conclusion}

In an attempt to develop oral liposomal delivery system, we used polymer-coating as means of improving liposomal properties. As our target was the delivery system for possible oral peptide and phytochemicals delivery, we used atenolol as a model molecule. As a prerequisite for successful delivery is the system's mucoadhesiveness, liposomes were coated with various polymers, namely, 
chitosan, Carbopol, and Eudragit L100 and Eudragit S100. The coating resulted in the increase in the original entrapment efficiency, as the coating was performed in the presence of unentrapped drug. Among tested polymers, coating with Eudragit S100 resulted in the highest entrapment and mucoadhesiveness. The consequence of coating was the increase in original liposomal size. The preliminary in vitro test of mucoadhesive properties of polymer-coated liposomes showed promising results, indicating that polymer-coated liposomes developed in this study may have a promising future in oral delivery of various molecules.

\section{Acknowledgments}

The authors are grateful to Lipoid $\mathrm{GmbH}$ for continuous generosity in providing phospholipids.

\section{Declaration of interest}

The authors report no conflict of interest. The authors alone are responsible for the content and writing of this paper.

\section{References}

1. Malik DK, Baboota S, Ahuja A, Hasan S, Ali J. (2007). Recent advances in protein and peptide drug delivery systems. Curr Drug Deliv, 4:141-51.

2. Stolnik S, Shakesheff K. (2009). Formulations for delivery of therapeutic proteins. Biotechnol Lett, 31:1-11.

3. Cui F, Qian F, Zhao Z, Yin L, Tang C, Yin C. (2009). Preparation, characterization, and oral delivery of insulin loaded carboxylated chitosan grafted poly(methyl methacrylate) nanoparticles. Biomacromolecules, 10:1253-58.

4. Andrews GP, Laverty TP, Jones DS. (2009). Mucoadhesive polymeric platforms for controlled drug delivery. Eur J Pharm Biopharm, 71:505-18.

5. Takeuchi H, Yamamoto KY. (2001). Mucoadhesive nanoparticulate systems for peptide drug delivery. Adv Drug Deliv Rev, 47:39-54.

6. Takeuchi H, Yamamoto H, Niwa T, Hino T, Kawashima Y. (1996). Enteral absorption of insulin in rats from mucoadhesive chitosan coated liposomes. Pharm Res, 13:896-901.

7. Drummond DC, Noble CO, Hayes ME, Park JW, Kirpotin DB. (2008). Pharmacokinetics and in vivo release rates in liposomal nanocarrier development. J Pharm Sci, 97:4696-740.

8. Iwanaga K, Ono S, Narioka K, Kakemi M, Morimoto K, Yamashita S, et al. (1999). Application of surface-coated liposomes for oral delivery of peptide: Effects of coating the liposome's surface on the GI transit of insulin. J Pharm Sci, 88:248-52.

9. Takeuchi H, Matsui Y, Yamamoto H, Kawashima Y. (2003). Mucoadhesive properties of carbopol or chitosan-coated liposomes and their effectiveness in the oral administration of calcitonin to rats. J Control Release, 86:235-42.
10. Filipovic-Grcic J, Skalko-Basnet N, Jalsenjak I. (2001). Mucoadhesive chitosan-coated liposomes: Characteristics and stability. J Microencapsul, 18:3-12.

11. Guo J, Ping Q, Jiang G, Huang L, Tong Y. (2003). Chitosan coated liposomes: Characterization and interaction with leuprolide. Int J Pharm, 260:167-73.

12. Mady MM, Darwish MM, Khalil S, Khalil WM. (2009). Biophysical studies on chitosan-coated liposomes. Eur Biophys J, 38:1127-33.

13. Werle M, Takeuchi H. (2009). Chitosan-aprotonin coated liposomes for oral peptide delivery: Development, characterization and in vivo evaluation. Int J Pharm, 370:26-32.

14. Muramatsu M, Kanada K, Nishida A, Ouchi K, Saito N, Yoshida $\mathrm{M}$, et al. (2000). Application of Carbopol to controlled release preparations I. Carbopol as a novel coating material. Int J Pharm, 99:77-83.

15. Singla AK, Chawla M, Singh A. (2000). Potential applications of carbomer in oral mucoadhesive controlled drug delivery system: A review. Drug Dev Ind Pharm, 26:913-24.

16. Kumar R, Gupta RB, Betageri GV. (2001). Formulation, characterization and in vitro release of glyburide from proliposomal beads. Drug Deliv, 8:25-7.

17. Lehr CM, Bouwstra JA, Schacht EH, Junginger HE. (1992). In vitro evaluation of mucoadhesive properties of chitosan and some other natural polymers. Int J Pharm, 78:43-8.

18. Sogias IA, Williams AC, Khutoryanskiy VV. (2008). Why is chitosan mucoadhesive? Biomacromolecules, 9:1837-42.

19. Baloum M, Friedman DI, Rubinstein A. (1997). Absorption enhancement of calcitonin in the rat intestine by carbopol-containing submicron emulsions. Int J Pharm, 154:235-43.

20. Jain D, Majumdar DK, Panda AK. (2006). Insulin loaded Eudragit L100 microspheres for oral delivery: Preliminary in vitro studies. J Biomater Appl, 21:195-211.

21. Jain D, Panda AK, Majumdar DK. (2005). Eudragit S100 entrapped insulin microspheres for oral delivery. AAPS PharmSciTech, 6:E100-7.

22. Galovic Rengel R, Barisic K, Pavelic Z, Zanic Grubisic T, Cepelak I, Filipovic-Grcic J. (2002). High efficiency entrapment of superoxidase dismutase into mucoadhesive chitosan-coated liposomes. Eur J Pharm Sci, 15:441-48.

23. de Abreu LRP, de Castro SAC, Pedrrazzoli J Jr. (2003). Atenolol quantification in human plasma by high-performance liquid chromatography: Application to bioequivalence study. AAPS PharmSci, 5:1-7.

24. Skalko N, Cajkovac M, Jalsenjak I. (1998). Liposomes with metronidazole for topical use: The choice of preparation method and vehicle. J Liposome Res, 8:283-93.

25. González-Rodríguez ML, Barros LB, Palma J, GonzálezRodríguez PL, Rabasco AM. (2007). Application of statistical experimental design to study the formulation variables influencing the coating process of lidocaine liposomes. Int J Pharm, 337:336-45.

26. Chowdary KPR, Rao YS. (2003). Design and in vitro and in vivo evaluation of mucoadhesive microcapsules of glipizide for oral controlled release: A technical note. Mucoadhesive microsphere for controlled drug delivery. AAPS PharmSciTech, 4:1-6.

27. Takeuchi H, Matsui Y, Sugihara H, Yamamoto H, Kawashima Y. (2005). Effectiveness of submicron-sized, chitosan-coated liposomes in oral administration of peptide drugs. Int J Pharm, 303:160-70.

28. Wu Z, Ping Q, Lai J. (2004). Hypoglycemic efficacy of chitosan coated insulin liposomes after oral administration in mice. Acta Pharmacol Sin, 25:966-72.

29. Shende P, Gaud R. (2009). Formulation and comparative characterization of chitosan, gelatin and chitosan-gelatin-coated liposomes of CPT-11-HCl. Drug Dev Ind Pharm, 35:612-18. 